\title{
Slow Prolonged Chewing or Mashing with Complete Re-Collection
}

National Cancer Institute

\section{Source}

National Cancer Institute. Slow Prolonged Chewing or Mashing with Complete Re-

Collection. NCI Thesaurus. Code C127199.

A finding of slow prolonged chewing or mashing with complete re-collection prior to swallowing. 